\title{
Mitigating Bunching with Bus-following Models and Bus-to-bus Cooperation
}

\author{
Konstantinos Ampountolas and Malcolm Kring \\ School of Engineering, University of Glasgow \\ Glasgow G12 8LT, United Kingdom \\ Email: Konstantinos.Ampountolas@glasgow.ac.uk, Malcolm.Kring@gmail.com
}

\begin{abstract}
Bus bunching is an instability problem where buses operating on high frequency public transport lines arrive at stops in bunches. In this work, we unveil that bus-following models can be used to design bus-to-bus cooperative control strategies and mitigate bunching. The use of bus-following models avoids the explicit modelling of bus-stops, which would render the resulting problem discrete, with events occurring at arbitrary time intervals. In a "follow-the-leader" two-bus system, bus-to-bus communication allows the driver of the following bus to observe (from a remote distance) the position and speed of a lead bus operating in the same transport line. The information transmitted from the lead bus is then used to control the speed of the follower to eliminate bunching. In this context, we first propose practical linear and nonlinear control laws to regulate space headways and speeds, which would lead to bunching cure. Then a combined state estimation and remote control scheme, which is based on the Linear-Quadratic Gaussian theory, is developed to capture the effect of bus stops, traffic disturbances, and randomness in passenger arrivals. To investigate the behaviour and performance of the developed approaches the 9-km 1-California line in San Francisco with about 50 arbitrary spaced bus stops is used. Simulations with real passenger data obtained from the San Francisco Municipal Transportation Agency are carried out. Results show bunching avoidance and significant improvements in terms of schedule reliability of bus services and delays. The proposed control is robust, scalable in terms of public transport network size, and thus easy to implement in real-world settings.
\end{abstract}

\section{INTRODUCTION}

It is well known from the 60's that due the passenger's demand variability at bus stops and speed heterogeneity, buses can randomly fall behind schedule [1]. In a two-bus transit system, if the lead bus in a line is delayed then the stops ahead of it will gather more waiting passengers, slowing it further (due to on-off passenger activity). Moreover, the following bus operating in the same line will pick up fewer passengers, causing its commercial speed to be higher than normal. Eventually, this positive feedback loop will result in the two buses meeting, with the lead bus having more passengers than the following bus. Thus, the two buses eventually pair up and travel as a single unit, although in reality overtaking is possible. This reduces the reliability of the system. Bus agencies often attempt to mitigate bus bunching by including slack time into their schedules and then asking bus drivers to be punctual (hold the bus up to a certain amount of time) at control points along the route. This cure is of limited effectiveness because the medicine (slack) is sometimes worse than the illness (irregular headways), see [2] and [3] for details.

Different control strategies have been proposed to mitigate bunching and improve the reliability of transit operations.
Basic schemes include patching and stop skipping. Patching is a scheme where buses are dispatched to fill gaps as they arise. This reactive to reliability problems method is obviously not optimal and it is used mainly in case of emergency. Stop skipping allows buses to recover from delays at the cost of reliability and customer satisfaction [4]. Schedule- or headwaybased holding strategies have several forms with varying levels of complexity (see e.g., [5], [6], [7], [3], [8]). The most basic version involves having a bus wait for an amount of time before continuing on the loop and it is therefore useful for both headway and schedule adherence. While holding can be effective at reducing bus bunching it reduces commercial speed [8]. Commercial speed and headway variance can be thought of as inversely proportional when a basic holding plan is in place and it is up to the bus agencies to find compromise between schedule reliability and commercial speed. Static holding involves fixed time buffers whereas dynamic holding responds to system events and adjusts buffers in real-time. However, static slack does not prevent large events from disrupting headways [7]. Dynamic holding can respond better to large headway disruptions. Recently [3], [8], [9] showed that dynamic holding strategies would increase commercial speed and return to equilibrium after large disruptions.

Technology has recently made much more nuanced and proactive control methods possible. A majority of what enables these new methods is the availability of real-time locational data. Modern buses are armed with surveillance and measurement equipment such as Automated Vehicle Location (AVL), Global Positioning (GPS) and Automated Passenger Counter (APC) devices, and telecommunications equipment to transmit information in real-time to traffic control centres. Thus it is possible to fully observe and communicate the true state of transit vehicles (speed, position, passenger load) operating in the same transit line in real-time. In this framework, the authors in [3] have proposed a cooperative, two-way-looking strategy based on the spacings in the front and back of each bus. To this end, a continuum idealisation of the bus bunching problem is proposed for which cooperative strategies can be designed. This model considers that bus stops are evenly spaced and bus delays due to on-off passenger activity are analogous to the number of passengers boarding the bus. In the same vein, the authors in [10] proposed a self-coordinating bus scheme where headways are dynamically self-equalising and the natural headway of the system tends to emerge spontaneously.

In this work, we propose and demonstrate that busfollowing (analogous to car-following) models can be used to design bus-to-bus cooperative control strategies and mitigate 
bunching. Car-following models are traditionally employed to describe the behaviour of closely spaced two-vehicle or stream of vehicles systems. The basic assumption of a followthe-leader model is that the following driver reacts to a stimulus from the lead car to maintain a specific headway distance between the two cars. This behaviour assumes driver's perception based on visual contact between the two cars and very short reaction times (which circumvents instabilities in case of stopped cars or stop-and-go phenomena). In a busfollowing model, the visual contact is missing because the two buses are far from each other. Moreover, a continuous-time bus-following model includes some simplification because it does not take into account the bus stops (where buses stop at arbitrary time intervals). We unveil that bus-to-bus cooperation and driver's response through real-time information permit the use of continuous-time bus-following models for bunching modelling and remote feedback control.

In the rest of the paper, we propose practical deterministic and stochastic bus-following models that can be used to mitigate bunching, remarkably without holding. Nonlinear and linear control laws are introduced to regulate space headways and speeds, which would lead to bunching cure. To capture the effect of bus stops, traffic disturbances, driver/motor errors and randomness in passenger arrivals, stochastic variables can be incorporated in a bus-following model. Thus a combined state estimation and control scheme is developed. To investigate the behaviour and performance of the proposed models, a simulation environment is developed, and simulations with real passenger data obtained from the San Francisco Municipal Transportation Agency are carried out. Results show bunching avoidance and significant improvements in terms of schedule reliability of bus services and delays.

\section{MODELling AND CONTROL BUNCHING WITH Bus-FOLLOWING MODELS}

Consider two buses $n$ (leader) and $n+1$ (follower) operating in the same public transport line. Assume that both buses are equipped with GPS devices reporting position $x(t)$ and speed $\dot{x}(t)=v(t)$ at any time $t$. Also that bus-tobus communication allows the driver of the following bus to observe the position and speed of his own bus and (from a remote distance) that of a lead bus operating in the same line. We call this class of "follow-the-leader" two-bus systems with a remote sensing capability as "bus-following models".

Fig. 1 illustrates the typical block diagram of a two-bus system with bus-to-bus cooperation. The kernel of the block diagram is the control strategy (or controller), whose task is to specify in real time the control inputs (e.g., acceleration or deceleration), based on available measurements (GPS and APC of the two-bus system), so as to achieve pre-specified goals (e.g., maintain a desired space headway) despite the influence of the various disturbances (observation, motor, and traffic). More precisely, GPS (and APC) information from the lead bus is transmitted to the following bus (with some noise). GPS signals can be corrupted unintentionally by external interfering sources such as tinted vehicle windows and buildings in urban canyons that block satellite transmission. Also APCs usually track every person who gets on or off the bus (including the operator) with some noise, which may lead to some irregularities in the data. The idea is to use the information transmitted from the lead bus to control the speed of the following bus in order to eliminate bunching. The driver of the following bus responds with a reaction time and a muscular response to control the speed. Speed control can be effectuated by a continuous adjustment of acceleration (or deceleration) of the following bus (via the acceleration and brake pedals), which is denoted $\ddot{x}(t)$ at time $t$. The applied control to bus dynamics is being corrupted by noise, including motor noise and traffic disturbances. Finally, the driver bus system includes a closed-loop structure to provide feedback. The feedback loop can inform the driver of the following bus when they are ahead or behind schedule (or have too small or too large a time or space headway) at all points along the route (by comparison of bus position and speed with the lead GPS data). Feedback can provide also information that may be useful for avoiding bunching by appropriate instructions (e.g., follow a desired speed that allows fast recovery).

\section{A. Deterministic Nonlinear and Linear Control Laws}

A first intuitive control law of a two-bus transit system considers that driver's response is proportional to the speed difference and the difference between their actual space headway and a desired (scheduled) headway given by

$$
\ddot{x}_{n+1}(t+T)=l_{1} \Delta \dot{x}_{n, n+1}(t)+l_{2}\left[\Delta x_{n, n+1}(t)-x^{d}\right]
$$

where $\Delta \dot{x}_{n, n+1}(t)=\dot{x}_{n}(t)-\dot{x}_{n+1}$ and $\Delta x_{n, n+1}(t)=x_{n}(t)$ $x_{n+1} ; T$ is the reaction time of the bus driver $n+1 ; l_{1}, l_{2}$ are control parameters; and $x^{d}$ is the desired space headway. The desired headway $x^{d}$ can be specified from the scheduled time headway of the corresponding bus line and an average operational speed. The choice of the control parameters $l_{1}$ and $l_{2}$ is performed via a trial-and-error procedure so as to achieve a satisfactory control behaviour for a given two-bus system; although optimised control gains could be determined by appropriate control methodologies as in Section II-B. If the two buses travel with the same speed the first term in (1) is negligible and acceleration (or deceleration) is based on the actual space headway of the two buses and a desired space headway. A similar control law was first proposed in [11] for car-following models and the only difference with (1) is that the reaction time $T$ of the driver-bus system is in general higher than of a driver-car system (which is approximately $1.5 \mathrm{~s}$ [12]). In a bus-following model the visual contact is missing (the two buses are far from each other) and reaction time depends on how often GPS locational data from the lead bus are transmitted to the following driver and the state of the following bus (traveling or stopped to drop-off or pick up passengers). Obviously the driver cannot react whenever the bus is stopped at bus stops.

A more complex nonlinear control law can be developed by assuming that driver's response is proportional to the velocity difference and inversely proportional to the difference between their actual space headway and a desired headway given by

$$
\ddot{x}_{n+1}(t+T)=\lambda \frac{\dot{x}_{n}(t)-\dot{x}_{n+1}(t)-v^{d}}{\left[x_{n}(t)-x_{n+1}(t)-x^{d}\right]^{2}}
$$

where $\lambda$ is a control gain and $v^{d}$ is an additional parameter. The correction term $v^{d}$ is introduced to circumvent instabilities whenever the lead bus is stopped for collecting passengers and the corresponding speed is virtually zero. Similar models (without considering a desired space headway and term $v^{d}$ ) for car-following models have been proposed in [13], [14]. 


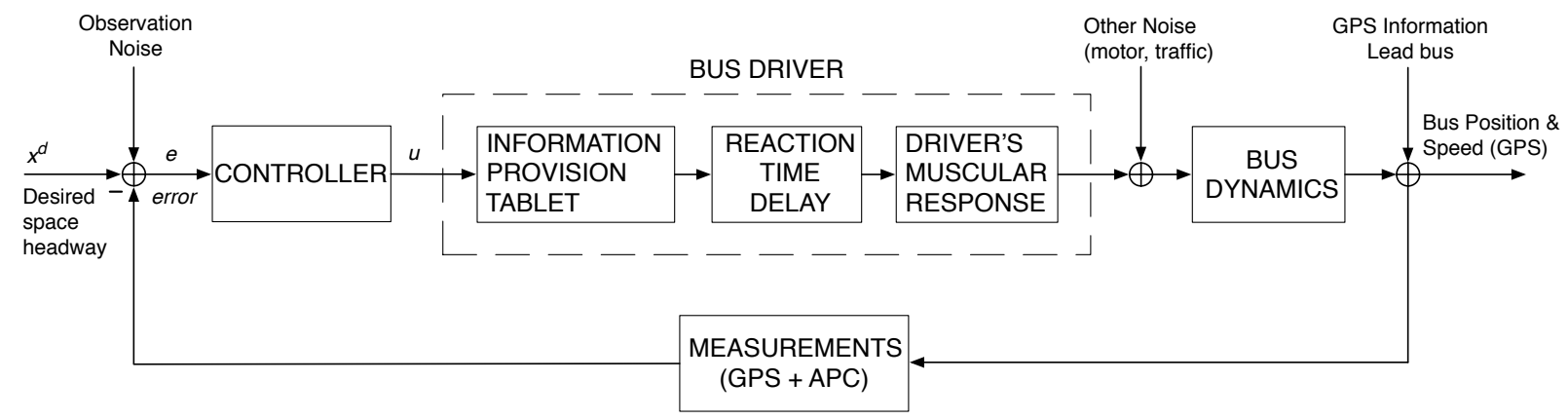

Fig. 1. Block diagram of the bus-following model with bus-to-bus cooperation.

\section{B. Stochastic Bus-following Models and Optimal Control}

Human operator behaviour for "follow-the-leader" models in presence of control being corrupted by noise has been studied extensively in the 60s and later on, see [15], [16], [17], [18], [19]. These models assume closely spaced twovehicle systems with basic vehicle motion dynamics, where each driver is required to track only the vehicle ahead. In the sequel, we propose a continuous-time bus-following model for bunching modelling that considers basic bus motion dynamics and uncertainties of different variables of the problem (bus stops, traffic disturbances, randomness in passenger arrivals). We then develop a rigorous combined state estimation and remote feedback control scheme based on Linear-Quadratic Gaussian (LQG) control [20], [21]. In fact, we unveil that the bus bunching problem can be viewed as a classic problem of relative space and speed regulation with noise in control theory.

Consider the bus-following model with bus-to-bus cooperation as in Fig. 1. The lead bus dynamics are represented by vehicle motion given by

$$
\begin{aligned}
& x_{n}(t+T)=a_{n, 1} x_{n}(t)+\varepsilon_{n, 1}(t) \\
& \dot{x}_{n}(t+T)=a_{n, 2} \dot{x}_{n}(t)+\varepsilon_{n, 2}(t)
\end{aligned}
$$

where $T$ is the reaction time, $x_{n}(t), \dot{x}_{n}(t)=v_{n}(t)$ are state variables, $a_{n, 1}, a_{n, 2}$ are constant modelling parameters and $\varepsilon_{n, 1}, \varepsilon_{n, 2}$ are zero-mean white gaussian noise with known variance. This linear model provides a mathematical description of the lead bus motion without considering any forces that affect the motion, i.e., a control term in (3)-(4) is absent. The basic idea of the bus-following model is to control the speed of the following bus (based on the location and speed of the leader) in order to eliminate bus bunching. Thus the follower bus dynamics are given by

$$
\begin{aligned}
& x_{n+1}(t+T)=a_{1} x_{n+1}(t)+b_{1} u_{n+1}(t)+\varepsilon_{n+1,1}(t) \\
& \dot{x}_{n+1}(t+T)=a_{2} \dot{x}_{n+1}(t)+b_{2} u_{n+1}(t)+\varepsilon_{n+1,2}(t)
\end{aligned}
$$

where $x_{n+1}(t), \dot{x}_{n+1}(t)=v_{n+1}(t)$ are state variables, $u_{n+1}(t)=\ddot{x}_{n+1}(t)$ is the control variable, $a_{1}, a_{2}, b_{1}, b_{2}$ are constant modelling parameters and $\varepsilon_{n+1,1}, \varepsilon_{n+1,2}$ are zeromean white gaussian noise with known variance. In the bus model (5)-(6), $u_{n+1}(t)$ represents the control applied by the driver (acceleration or deceleration) via the acceleration and brake pedals. The stochastic variables $\varepsilon_{n+i, j}$, for all $i=0,1$, $j=1,2$ are introduced to capture the effect of bus stops, traffic disturbances, and randomness in passenger arrivals.

Expressions (3)-(6) provide a continuous-time busfollowing model that does not allow for direct consideration of the bus stops (where buses stop at arbitrary time intervals) and passenger loads. Nevertheless, uncertainty and variability of different variables of the problem can be captured by the noise terms in (3)-(6). The number of bus stops (fixed and known) and historical (or fusion with real-time) data of passenger activity for a specific line can reflect the typical time variation of passenger arrivals. In addition, traffic and driver/motor noise can be obtained with different sensors in real-time. This is the price to pay for avoiding the explicit modelling of bus-stops which would render the resulting problem discrete (Discrete Event Dynamic System) and would lead to complex models difficult to use for control purposes without relaxations.

Expressions (3)-(6) can be combined into a discrete-time ${ }^{1}$ linear system with noise given by

$$
\mathbf{x}(k+1)=\mathbf{A} \mathbf{x}(k)+\mathbf{B} u(k)+\gamma(k)
$$

where $\mathbf{x} \in \mathbb{R}^{4 \times 1}$ (with elements $x_{n+i, j}, \dot{x}_{n+i, j}$ for all $i=0,1$, $j=1,2$ ) is the state vector of both leader and follower position and velocity, and $u \equiv u_{n+1}$ is the control; $\mathbf{A} \in \mathbb{R}^{4 \times 4}$ (with elements $a_{n+i, j}$, for all $i=0,1, j=1,2$ ) and $\mathbf{B} \in \mathbb{R}^{4 \times 1}$ (with elements $\left.b_{n+1, i}, i=1,2\right)$ are the state and control matrices, respectively; $\gamma \in \mathbb{R}^{4}$ (with elements $\varepsilon_{n+i, j}$, for all $i=0,1$, $j=1,2$ ) is a Gaussian noise process with known covariance matrix $\Gamma$ given by

$$
\mathbf{E}\{\gamma(k)\}=\mathbf{0}, \quad \mathbf{E}\left\{\gamma\left(k_{i}\right) \cdot \boldsymbol{\gamma}^{T}\left(k_{j}\right)\right\}=\boldsymbol{\Gamma} \cdot \delta_{i j}, \quad \boldsymbol{\Gamma} \succeq 0
$$

where $\delta$ is the Kronecker delta function. We also assume that the initial state $\mathbf{x}(0)$ has a known mean $\mathbf{x}_{0}$ and covariance matrix $\Pi_{0}$ given by

$$
\mathbf{E}\{\mathbf{x}(0)\}=\mathbf{x}_{0}, \quad \mathbf{E}\left\{\left[\mathbf{x}(0)-\mathbf{x}_{0}\right] \cdot\left[\mathbf{x}(0)-\mathbf{x}_{0}\right]^{T}\right\}=\mathbf{\Pi}_{0} .
$$

The system output vector $\mathbf{y} \in \mathbb{R}^{2}$ for the augmented system (7) is given by

$$
\mathbf{y}(k)=\mathbf{H x}(k)+\boldsymbol{\zeta}(k)
$$

where $\boldsymbol{\zeta}$ is the observation noise and $\mathbf{H}$ is the observation matrix consisting of 0 's and 1's. More precisely, $\mathbf{H}$ matrix allows the lead bus state variables to be compared with those of the following bus. An appropriate choice is

$$
\mathbf{H}=\left[\begin{array}{cccc}
1 & 0 & -1 & -\alpha h^{d} \\
0 & 1 & 0 & -1
\end{array}\right]
$$

\footnotetext{
${ }^{1}$ For control implementation purposes, we define a discrete time index $k$ such that $t=k T$ with $T$ the sampling period (reaction time) and denote
} $x(k)=x(k T)$ analogously for all variables. 
where $\alpha$ is a constant parameter and $h^{d}$ is a desired (scheduled) time headway between two buses, which is known from the timetable. Parameter $\alpha$ is introduced to capture the correlation between actual space headway $x_{n}-x_{n+1}$ and a desired headway $x^{d}$, where $x^{d}$ is assumed to be a linear function of the following bus velocity $\dot{x}_{n+1}$ and the desired time headway $h^{d}$, i.e. $x^{d} \propto \alpha \dot{x}_{n+1}(t) h^{d}$. Note that in general, the desired spacing is a nonlinear function of the velocity of the following bus, time headway, passenger load and traffic conditions in the transit line. Noise vector $\zeta \in \mathbb{R}^{2}$ is a Gaussian noise process with known covariance matrix $\mathbf{Z}$ given by

$$
\mathbf{E}\{\boldsymbol{\zeta}(k)\}=\mathbf{0}, \quad \mathbf{E}\left\{\boldsymbol{\zeta}\left(k_{i}\right) \cdot \boldsymbol{\zeta}^{T}\left(k_{j}\right)\right\}=\mathbf{Z} \cdot \delta_{i j}, \quad \mathbf{Z} \succ 0 .
$$

In addition, we assume that the system (7)-(8) is reachable and observable; and the initial state condition $\mathbf{x}_{0}$ is uncorrelated with the input noise $\gamma(k)$ and observation noise $\boldsymbol{\zeta}(k)$, i.e., $\mathbf{E}\left\{\boldsymbol{\gamma}(k) \cdot \mathbf{x}_{0}^{T}\right\}=\mathbf{E}\left\{\boldsymbol{\zeta}(k) \cdot \mathbf{x}_{0}^{T}\right\}=\mathbf{0}$. Finally both noise inputs are assumed uncorrelated (although can be correlated), i.e., $\mathbf{E}\left\{\gamma(k) \cdot \boldsymbol{\zeta}^{T}(k)\right\}=\mathbf{0}$.

Our control objective is to regulate the speed of the following bus with minimum effort so as to maintain the actual space headway of a two-bus system to a desired pre-specified headway, and as a consequence to avoid bunching. A quadratic criterion that considers this control objective has the form

$$
\begin{aligned}
\mathcal{L}=\mathbf{E}\left\{\sum _ { k = 0 } ^ { \infty } \left\{q_{1}\left[x_{n}(k)-x_{n+1}(k)-x^{d}\right]^{2}+\right.\right. \\
\left.\left.q_{2}\left[\dot{x}_{n}(k)-\dot{x}_{n+1}(k)\right]^{2}+r u(k)^{2}\right\}\right\}
\end{aligned}
$$

where $q_{1} \geq 0, q_{2} \geq 0$ and $r>0$ are weighting constants. The first term in (10) is responsible for the minimisation of deviations of the space headway from a desired spacing. The second term is responsible for normalising the speeds between the two buses. Note that an additional correction term $v^{d}$ (cf. (2)) may be introduced in the second term to circumvent speed instabilities whenever the lead bus is stopped for collecting passengers and the corresponding speed is virtually zero. Clearly the third term is responsible for avoiding bunching with minimum control effort. Weights $q_{1}, q_{2}$ must be chosen such that the corresponding subgoals are satisfied. More precisely, $q_{1}=1 / x^{d}$ and $q_{2}=1 / v^{c}$ are appropriate values, where $v^{c}$ is the commercial speed. Commercial speed is defined as the average operational speed of the buses, including cruising, passenger alighting and boarding. Weight $r$ influences the magnitude of the control reactions and is selected through a trial-and-error procedure so as to achieve a satisfactory control behaviour for a given application transport line. The infinite time horizon in (10) is taken in order to obtain a time-invariant feedback law according to the LQG control theory.

To design the combined state estimation and control scheme, we assume at time $k>k_{0}$ availability of portable information

$$
\mathcal{I}(k)=\left\{\mathbf{y}\left(k_{0}\right), u\left(k_{0}\right), \mathbf{y}\left(k_{0}+1\right), u\left(k_{0}+1\right), \ldots, \mathbf{y}(k)\right\} .
$$

According to the LQG theory [20], [21] an appropriate control law has the form

$$
u(k)=-\mathbf{L} \hat{\mathbf{x}}(k)
$$

where $\mathbf{L} \in \mathbb{R}^{1 \times 4}$ (depends on $\mathbf{A}, \mathbf{B}$ and $q_{1}, q_{2}, r$ ) is a timeinvariant control gain, which is calculated by the solution of the corresponding deterministic Linear-Quadratic (LQR) control problem (not shown); and $\hat{\mathbf{x}}(k)$ is the output of the optimal state estimator, i.e. Kalman Filter. The estimate $\hat{\mathbf{x}}(k)$ is generated in real-time by the recursive estimator

$$
\hat{\mathbf{x}}(k)=\mathbf{A} \hat{\mathbf{x}}(k-1)+\mathbf{B} u(k-1)+\mathbf{K}[\mathbf{y}(k-1)-\mathbf{H} \hat{\mathbf{x}}(k-1)]
$$

where $\hat{\mathbf{x}}(0)=\mathbf{x}_{0}$ is known and $\mathbf{K} \in \mathbb{R}^{4 \times 2}$ (depends on $\mathbf{A}, \mathbf{H}$ and $\boldsymbol{\Gamma}, \mathbf{Z}$ ) is a time-invariant (although might be timevariant) estimator gain, which is calculated by a recursive Riccati equation (not shown) according to the Kalman Filter theory. The Separation Theorem guarantees that the overall LQG control design is optimal in the sense that the control law (11) minimises the cost criterion (10). The stability of control law (11) may be mathematically guaranteed under certain assumptions (presence of white noise disturbances).

The final control law reads

$$
u(k)=l_{1} \Delta \hat{\dot{x}}_{n, n+1}(k)+l_{2}\left[\Delta \hat{x}_{n, n+1}(k)-l_{3} \hat{\dot{x}}_{n+1}(k)\right]
$$

where $l_{1}, l_{2}$ and $l_{3}$ are elements of matrix $\mathbf{L}$ (calculations not shown). Note that (13) is similar to (1) with $l_{3}=0$ and deterministic state vector, which corresponds to the standard LQR solution without the Kalman Filter. The real-time application of the LQG scheme calls for estimates of $\hat{\mathbf{x}}(k)$ via (12) (starting from known $\hat{\mathbf{x}}_{0}$ ) based on which the scheme executes the control law (13) and returns the control (acceleration or deceleration) for application to the driver. Thus the required calculations in real-time are limited to the parallel execution of (12)-(13), while the control and estimation gains $\mathbf{L}$ and $\mathbf{K}$ are calculated off-line (although may be time-variant and updated on-line). Finally, it should be noted that the bus-following model given by (7) is generic and could be applied to public transport lines with arbitrary geometry and characteristics.

\section{APPLICATION AND RESUlTS}

\section{A. Simulation Environment}

A simulation environment was designed in Matlab to reflect real world bus lines including arbitrary placed stops and onoff passenger activity. The simulation environment emulates a closed-loop bus line and includes a depot where buses leave the depot at specified intervals (bus frequency) and return to the depot when they complete one loop. Overtaking is allowed in case of bunching as in reality. Variability is introduced via randomness in passenger arrivals at bus stops. The probability of arrival per time-unit is variable with time and between different bus stops. This emulates variant passenger behaviour in time and space, e.g. rush hour. In utilising real world data, the stop locations, speed limits, schedule, bus capacity, and passenger behaviour are accounted for and, when applicable, are time-variant. The simulator is stochastic, i.e., the same seed is initialised with different generators so that different replications to produce different results for the same demand scenario. It also assumes that delays at bus stops due to on-off passenger activity are analogous to the number of passengers alighting and boarding the bus. Alighting and boarding times of the passengers are set to 1-3 s and 2-5 s, respectively. In case that real passenger activity data at stops are not available, the number of people getting off at any stop is a random number between 0 and the number of people at the bus. In 


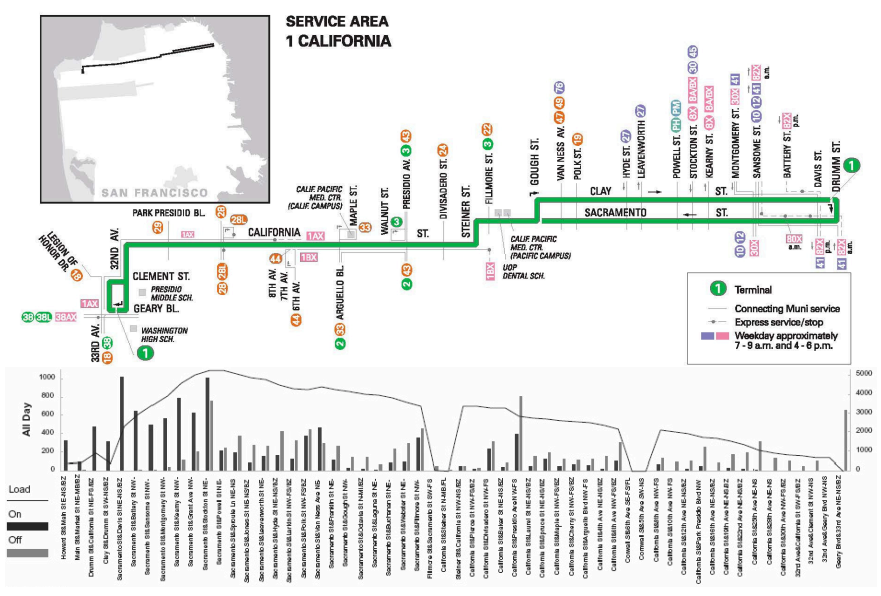

Fig. 2. The service area of 1-CALIFORNIA line and passenger activity graph. Left $y$-axis is the scale for the on-off activity at each bus stop; Right $y$-axis is the scale for the solid line showing total load; Solid line indicates the total number of passengers that ride through stops; The bars are the number of passengers that get on (shown in black) or off (shown in gray) at bus stops.

addition, a random bounded time delay is added to simulate buses slowing to a stop and accelerating to operating speed after stopping. The simulator includes an Application Program Interface (API) that allows the simulator to interact in real-time with an external program (control strategy), exchanging data, pass inputs (control) and receive outputs (position, velocity, on-off passenger activity). At regular intervals headway data, state and control trajectories are gathered for analysis.

\section{B. Experimental Data and Results}

To investigate the efficiency of the developed approaches to the problem of bus bunching, two different public transport lines are considered. The first case is a $2-\mathrm{km}$ closed-loop transport line with 10 arbitrary spaced bus stops. The second case is the 9-km 1-California line in San Francisco (service area of California St, Clay St and Sacramento St) with about 50 arbitrary spaced bus stops, depicted in Fig. 2. For each line we apply and compare the behaviour of the control laws given by (2) (denote it "Lambda") and (1) (denote it "LQR") with a no control case wherein buses operating with a nominal speed.

To fine tune the two controllers different passenger demand scenarios were used and the simulation environment outlined in the previous section was employed. A fine tuning procedure (trial-and-error) involves the suitable choice of the control gains $l_{1}, l_{2}$, and $\lambda$ so as to achieve a satisfactory control behaviour and performance with respect to schedule adherence and reliability of bus service. Initially the $2-\mathrm{km}$ line was used to investigate the behaviour of the deterministic control laws for the bus-following model with only two buses operating in the same line, for different demand scenarios with time horizon of $2 \mathrm{~h}$. The most satisfactory results with respect to schedule adherence, bunching avoidance, and reliability of bus service were obtained with control gains $\left(l_{1}, l_{2}\right)=(0.075,2.5)$ for the control law (1), and $\lambda=0.005$ for the control law (2). The optimal solution for control law (13) is very close to $\left(l_{1}, l_{2}\right)=(0.075,2.5)$ and can be obtained by the LQG theory with $q_{1}=1 / x^{d}, q_{2}=1 / v^{c}$ and $r=10^{-4}$, where commercial speed $v^{c}=10 \mathrm{~km} / \mathrm{h}$, desired time headway $h^{d}=7$ $\min$ and $x^{d} \propto \alpha \dot{x}_{n+1}(t) h^{d}=\alpha v^{c} h^{d}(\alpha$ unit correction parameter). Then tests for the 1-California line were conducted with real passenger (on-off activity) data obtained from the San
TABLE I. PERformance Indices For the 1-CALIFornia Line

\begin{tabular}{l||c|c}
\hline Control Method & Commercial Speed $(\mathrm{km} / \mathrm{h})$ & Headway Adherence \\
\hline No Control & 14.6 & 0.4282 \\
Lambda & 13.8 & 0.3079 \\
LQR & 12.6 & 0.1794
\end{tabular}

Francisco Municipal Transportation Agency (SFMTA), see the caption in Fig. 2 for details on the data format. SFMTA data [22] were available for specific time periods (e.g., AM peak, PM peak, Midday, School, etc.) as well as for a whole day (aggregated as in Fig. 2). Therefore tests were conducted for different peak periods (with a time horizon of 3-4 hours) and a whole day. For all reported results the reaction time $T$ of the bus driver is set to $30 \mathrm{~s}(10 \mathrm{~s}$ and $60 \mathrm{~s}$ were also tested), this includes GPS latency and delays due to stops.

Table I displays the performance of the three control methods for the 1-California line in terms of commercial speed (in $\mathrm{km} / \mathrm{h}$ ) and headway adherence. Headway adherence is defined as the ratio of standard deviation of headway deviation (difference between actual and scheduled headway) over scheduled headway, i.e. $\operatorname{std}\left(h-h^{d}\right) / h^{d}$ where $h$ and $h^{d}$ are the actual and scheduled headways, respectively. The headway adherence provides insights for the reliability of the bus lines. A value close to 0 indicates a small deviation from the scheduled headway and thus high reliability of bus services. On the other hand, high values of headway adherence results to high waiting times for the passengers at bus stops. As can be seen in Table I the no control case has the highest commercial speed among the three control methods because no control is applied to buses, as expected. However, the lower commercial speed in case of control (due to acceleration and deceleration) proves beneficial for the reliability of bus services. Thus, the ranking of the control methods with respect to headway adherence is the opposite. As can be seen, both Lambda and LQR lead to a reduction of headway adherence and waiting times for the passengers (not shown) compared to no control. The LQR control exhibits the best reliability among all strategies, while it is the slowest (slower is faster effect, i.e., better reliability and customer satisfaction).

Fig. 3 presents the results obtained from the application of the three control methods to the 1-California line for an AM peak (real data) scenario with scheduled headway 7 min (420 s). Figs 3(a)-(c) depict the obtained trajectories of a stream of buses operating in line 1. In Figs 3(b)-(c) buses have been controlled in pairs with a follow-the-leader like model and bus-to-bus cooperation, as in Fig. 1. Circles on the trajectories indicate the control points, thus circles disappear the time after a lead bus reach its destination (obviously no circles present on the trajectory of the first bus in the line). As can be seen, bus bunching is observed under no control (Fig. 3(a)) while it is avoided with control. LQR is seen to perform better from Lambda control (cf. Fig. 3(b) with Fig. 3(c)).

Figs 3(d)-(f) display the headway distributions under the three control methods (headways collected every $1 \mathrm{~s}$ ). A larger concentration of values around the scheduled headway (420 s) indicates a smaller deviation from the schedule and higher reliability. As can be seen from the distributions, LQR (mean $419 \mathrm{~s}$, std $58 \mathrm{~s}$ ) exhibits the best performance followed by Lambda (mean $412 \mathrm{~s}$, std $128 \mathrm{~s}$ ) and no control (mean 411 $\mathrm{s}$, std $170 \mathrm{~s}$ ). The ranking of the strategies with respect to headways is in agreement with the findings in Table I (see schedule adherence and reliability of bus service). 


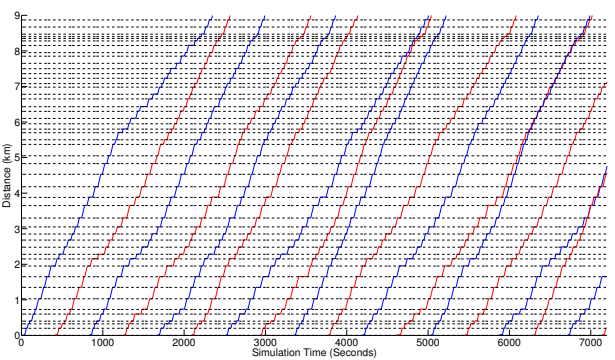

(a)

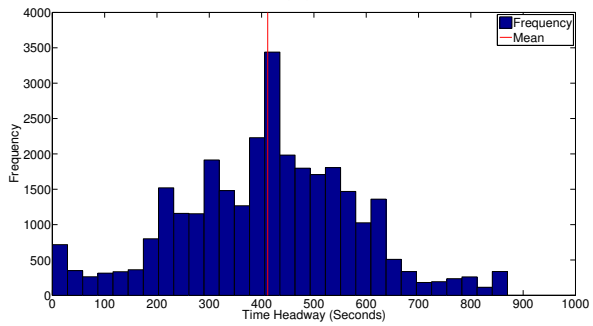

(d)

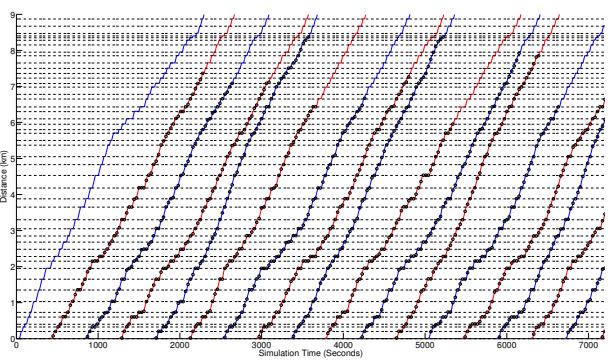

(b)

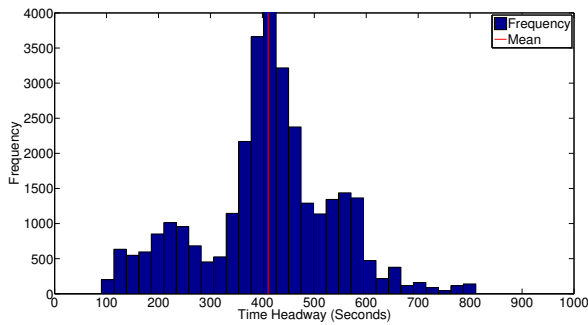

(e)

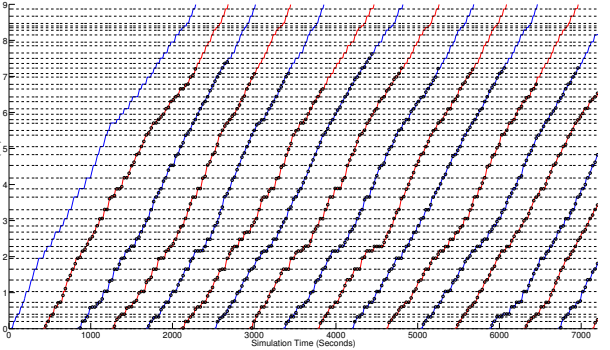

(c)

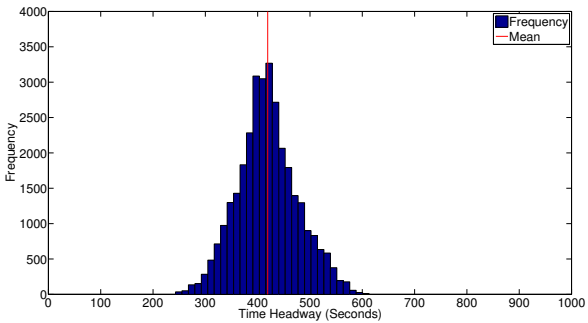

(f)

Fig. 3. (a)-(c) Time-space diagrams with trajectories from a stream of buses operating in the same line under no control, Lambda (see (2)) and LQR (see (1)) control, respectively; Horizontal dotted lines indicate the location of bus stops; Circles indicate points of control activation. (d)-(f) Bus headway distribution under no control, Lambda (see (2)) and LQR (see (1)) control, respectively.

\section{CONCLUSIONS}

The paper addressed the problem of bus bunching in public transport lines. The presented methodological framework combines bus-following models and bus-to-bus cooperative control strategies. The use of bus-following models avoids the explicit modelling of bus-stops which would render the resulting problem discrete, with events occurring at arbitrary time intervals. We unveiled that the bunching problem can be viewed as a classical regulation problem in control theory. To this end, we proposed practical linear and nonlinear control laws to regulate space headways and speeds, which would lead to bunching cure. A combined state estimation and remote control scheme was also developed. Results from the application of the proposed control laws to the 9-km 1-California line in San Francisco with about 50 arbitrary spaced bus stops showed bunching avoidance and significant improvements in terms of schedule reliability of bus services and delays. The proposed control laws are easy to implement and can be used for realtime bunching control in real-life settings. For implementation, a tablet or other external device can be utilised.

\section{REFERENCES}

[1] G. F. Newell and R. B. Potts, "Maintaining a bus schedule," in Proc. of the 2nd Australian Road Research Board, vol. 2, Melbourne, Australia, 1964, pp. 388-393.

[2] C. F. Daganzo, Fundamentals of Transportation and Traffic Operations. New York, NY: Pergamon Press, 1997.

[3] C. F. Daganzo and J. Pilachowski, "Reducing bunching with bus-to-bus cooperation," Transport Res Part B, vol. 45, no. 1, pp. 267-277, 2011.

[4] A. Sun and M. Hickman, "The real-time stop-skipping problem," Journal of Intell Transport Systems, vol. 9, no. 2, pp. 91-109, 2005.

[5] G. F. Newell, "Control of pairing of vehicles on a public transportation route, two vehicles, one control point," Transportation Science, vol. 8, no. 3, pp. 248-264, 1974.

[6] X. J. Eberlein, N. H. M. Wilson, and D. Bernstein, "The holding problem with real-time information available," Transportation Science, vol. 35 , no. 1 , pp. $1-18,2001$.

[7] C. F. Daganzo, "A headway-based approach to eliminate bus bunching: Systematic analysis and comparisons," Transportation Research Part B, vol. 43, no. 10, pp. 913-921, 2009.
[8] Y. Xuan, J. Argote, and C. F. Daganzo, "Dynamic bus holding strategies for schedule reliability: Optimal linear control and performance analysis," Transportation Research Part B, vol. 45, no. 10, pp. 1831-1845, 2011.

[9] Y. Ji and H. M. Zhang, "Dynamic holding strategy to prevent buses from bunching," Transport Research Record, vol. 2352, pp. 94-103, 2013.

[10] J. J. Bartholdi III and D. D. Eisenstein, "A self-coordinating bus route to resist bus bunching," Transportation Research Part B, vol. 46, no. 4, pp. 481-491, 2012.

[11] W. Helly, "Simulation of bottlenecks in single-lane traffic flow," in Proc. Symposium on the Theory of Traffic Flow, R. Herman, Ed., Elsevier, Amsterdam, The Netherlands, 1961, pp. 207-238.

[12] R. E. Chandler, R. Herman, and E. W. Montroll, "Traffic dynamics: Studies in car following," Operations Research, vol. 6, no. 2, pp. 165184, 1958.

[13] L. A. Pipes, "An operational analysis of traffic dynamics," Journal of Applied Physics, vol. 24, no. 3, pp. 274-281, 1953.

[14] D. C. Gazis, R. Herman, and R. B. Potts, "Car-following theory of steady-state traffic flow," Operations Research, vol. 7, no. 4, pp. 499$505,1959$.

[15] J. Tyler, J., "The characteristics of model-following systems as synthesized by optimal control," IEEE Transactions on Automatic Control, vol. 9, no. 4, pp. 485-498, 1964.

[16] D. Kleinman, S. Baron, and W. Levison, "An optimal control model of human response Part I: Theory and validation," Automatica, vol. 6, no. 3, pp. 357-369, 1970.

[17] S. Baron, D. Kleinman, and W. Levison, "An optimal control model of human response Part II: Prediction of human performance in a complex task," Automatica, vol. 6, no. 3, pp. 371-383, 1970.

[18] G. A. Bekey, G. O. Burnham, and J. Seo, "Control theoretic models of human drivers in car following," Human Factors: The Journal of the Human Factors and Ergonomics Soc, vol. 19, no. 4, pp. 399-413, 1977.

[19] A. Adamski and A. Turnau, "Simulation support tool for real-time dispatching control in public transport," Transportation Research Part A, vol. 32, no. 2, pp. 73-87, 1998.

[20] K. J. Åström, Introduction to Stochastic Control Theory. New York, NY: Academic Press, 1970.

[21] M. Athans, "The discrete time Linear-Quadratic-Gaussian stochastic control problem," Annals of Economic and Social Measurement, vol. 1, no. 4, pp. 449-491, 1972.

[22] SFMTA, http://sfmta.com/, [Online; accessed June-2014]. 